\title{
$\left\{\left(\mathrm{Me}_{2} \mathrm{NH}_{2}\right)_{2}\left[\mathrm{Fe}_{2}(\mathrm{ox})_{2} \mathrm{Cl}_{4}\right] \cdot \mathrm{H}_{2} \mathrm{O}\right\}_{n}(\mathrm{ox}=$ oxalate $)$ 中客体水分子诱导的 介电弛豫行为
}

\author{
王宾 ${ }^{\dagger}$ 唐雯 ${ }^{\dagger}$ 赵海霞* 龙腊生* 郑兰䔉 \\ (厦门大学 化学化工学院化学系 固体表面物理化学国家重点实验室 能源材料化学协同创新中心 厦门 361005)
}

\begin{abstract}
摘要 以双齿配位的草酸根为配体和 $\mathrm{FeCl}_{3}$ 反应得到了化合物 $\left\{\left(\mathrm{Me}_{2} \mathrm{NH}_{2}\right)_{2}\left[\mathrm{Fe}_{2}(\mathrm{ox})_{2} \mathrm{Cl}_{4}\right] \cdot \mathrm{H}_{2} \mathrm{O}\right\}_{n}$ (ox $=$ oxalate) $\left(\mathbf{1} \cdot \mathrm{H}_{2} \mathrm{O}\right)$. 在 真空和加热的条件下, $\mathbf{1} \cdot \mathrm{H}_{2} \mathrm{O}$ 脱水发生单晶到单晶的转变, 得到了化合物 $\left\{\left(\mathrm{Me}_{2} \mathrm{NH}_{2}\right)_{2}\left[\mathrm{Fe}_{2}(\mathrm{ox})_{2} \mathrm{Cl}_{4}\right]\right\}_{n}$ (1). 磁性研究表明 客体水分子存在与否对化合物磁相互作用没有明显影响. 介电性质研究表明客体水分子的存在可影响化合物中客体胺 和氢原子的动态驰豫过程. 化合物 $1 \cdot \mathrm{H}_{2} \mathrm{O}$ 在 $250 \mathrm{~K}, 80 \mathrm{~K}$ 和 $50 \mathrm{~K}$ 呈现三个介电弛豫过程，而化合物 $\mathbf{1}$ 仅在 $80 \mathrm{~K}$ 表现出 单一弛豫过程.
\end{abstract}

关键词 介电驰豫; 单晶-单晶转化; 水; 磁性

\section{Dielectric Relaxation Triggered by Guest Water Molecule Based on the $\left\{\left(\mathrm{Me}_{2} \mathrm{NH}_{2}\right)_{2}\left[\mathrm{Fe}_{2}(\mathrm{ox})_{2} \mathrm{Cl}_{4}\right] \cdot \mathrm{H}_{2} \mathrm{O}\right\}_{n}$ (ox = oxalate)}

\author{
Wang, Bin $^{\dagger}$ \\ Tang, Wen ${ }^{\dagger}$ \\ Zhao, Haixia* \\ Long, Lasheng* \\ Zheng, Lansun
}

(Collaborative Innovation Center of Chemistry for Energy Materials, State Key Laboratory of Physical Chemistry of Solid Surfaces and Department of Chemistry, College of Chemistry and Chemical Engineering, Xiamen University, Xiamen 361005, China)

\begin{abstract}
It is well known that water is one of the most important chemical constituent in the nature, and thus is regarded as the genesis of life. Meanwhile, water plays an irreplaceable role in biology process, such as regulating the affinity between fatty acids and alkyl chains and controlling the conformation of DNA. In the crystal chemistry, water molecules in compounds will also significantly affect its structure, physical and chemical properties. Among them, single crystal to single crystal (SCSC) transformations induced by water molecules have attracted great attention, because those transformations often change the physical properties such as magnetic, dielectric and proton conduction. More importantly, it is facile to obtain detailed structural information, which leads to more directly and better understanding the transformation mechanism and structure-properties relationship. In this work, we report the SCSC transformation in the compound of $\left\{\left(\mathrm{Me}_{2} \mathrm{NH}_{2}\right)_{2}-\right.$ $\left.\left[\mathrm{Fe}_{2}(\mathrm{ox})_{2} \mathrm{Cl}_{4}\right] \cdot \mathrm{H}_{2} \mathrm{O}\right\}_{n}(\mathrm{ox}=$ oxalate $)\left(\mathbf{1} \cdot \mathrm{H}_{2} \mathrm{O}\right)$, which was prepared through the hydrothermal reaction of oxalate and $\mathrm{FeCl}_{3}$ in the presence of dimethylamine chloride. Under vacuum and heating conditions, $\mathbf{1} \cdot \mathrm{H}_{2} \mathrm{O}$ underwent SCSC transformations, formed compound $\mathbf{1} \cdot \mathbf{1} \cdot \mathrm{H}_{2} \mathrm{O}$ and $\mathbf{1}$ were characterized by X-ray single-crystal diffraction, powder X-ray diffraction, magnetic, dielectric and thermogravimetric analysis. Magnetic studies on $\mathbf{1} \cdot \mathrm{H}_{2} \mathrm{O}$ and $\mathbf{1}$ show that the presence or absence of guest water molecules had no significant effect on their magnetic interaction. Investigation on the dielectric properties of $\mathbf{1} \cdot \mathrm{H}_{2} \mathrm{O}$ and $\mathbf{1}$ reveals that the presence of guest water molecules can influence the dynamic relaxation process of guest amine and water in the compound. Compound $\mathbf{1} \cdot \mathrm{H}_{2} \mathrm{O}$ exhibited three dielectric relaxations at $250 \mathrm{~K}, 80 \mathrm{~K}$ and $50 \mathrm{~K}$ respectively, while 1 only showed a single relaxation process at $80 \mathrm{~K}$. This work sheds light on the further exploration of SCSC transformations associated with the dielectric relaxation.

Keywords dielectric relaxation; SCSC structural transformation; water; magnetism
\end{abstract}

\section{1 引言}

水是自然界中最重要的物质之一, 并在生物体内有 着无可替代的重要作用, 如调节脂肪酸与烷基链的亲和 力, 控制 DNA 的构象等 ${ }^{[-5]}$. 在晶体化学中, 水分子的 存在与否同样会显著影响化合物的结构、物理和化学性
质，如单晶-单晶转变、磁性、介电性、质子传导等 ${ }^{[6-10]}$. 其中, 客体水分子缺失导致的单晶-单晶转变由于能够 通过测试得到转变前后的详细结构信息, 对理解和揭示 水分子影响化合物结构和性质之间的关系至关重 要 ${ }^{[1-15]}$.

目前关于客体水分子缺失导致的单晶-单晶转变的

*E-mail: hxzhao@xmu.edu.cn; lslong@xmu.edu.cn

$\uparrow$ These authors contributed equally to this work

Received August 29, 2020; published October 19, 2020.

Supporting information for this article is available free of charge via the Internet at http://sioc-journal.cn.

Project supported by the National Natural Science Foundation of China (Nos. 21431005, 21721001, 21571150).

项目受国家自然科学基金(Nos. 21431005, 21721001, 21571150)资助. 
研究主要集中在磁性和晶体结构方面 ${ }^{[16-21]}$. 由于晶体中 水分子的缺失往往导致由晶体内部水分子氢键网络的 破坏, 在这类单晶-单晶转变过程中, 通常会伴随着电 学性质的改变. 这样, 人们通过测试单晶-单晶转变前 后化合物的介电常数、介电损耗 ${ }^{[22-23]}$, 并结合单晶-单晶 转变前后化合物的结构信息, 可望揭示客体水分子对其 介电性质影响的本质. 然而, 迄今为止单晶-单晶转变 所伴随的介电及铁电性质变化的研究仍较为少见 ${ }^{[24-25]}$.

本工作中, 我们采用草酸根为配体和 $\mathrm{FeCl}_{3}$ 反应得 到了化合物 $\left\{\left(\mathrm{Me}_{2} \mathrm{NH}_{2}\right)_{2}\left[\mathrm{Fe}_{2}(\mathrm{ox})_{2} \mathrm{Cl}_{4}\right] \cdot \mathrm{H}_{2} \mathrm{O}\right\}_{n} \quad(\mathrm{ox}=$ oxalate) $\left(\mathbf{1} \cdot \mathrm{H}_{2} \mathrm{O}\right)$. 在真空和加热的条件 $1 \cdot \mathrm{H}_{2} \mathrm{O}$ 发生了单 晶到单晶转变, 得到了化合物 $\left\{\left(\mathrm{Me}_{2} \mathrm{NH}_{2}\right)_{2}\left[\mathrm{Fe}_{2}(\mathrm{ox})_{2} \mathrm{Cl}_{4}\right]\right\}_{n}$ (1). 磁性研究表明客体水分子存在与否对化合物磁相 互作用没有明显影响，而客体水分子的存在诱导出现了 介电弛豫过程, 化合物 $1 \cdot \mathrm{H}_{2} \mathrm{O}$ 在 $250 \mathrm{~K}, 80 \mathrm{~K}$ 和 $50 \mathrm{~K}$ 出 现了三个介电弛豫过程, 随着水分子的脱去, 化合物 $\mathbf{1}$ 仅在 $80 \mathrm{~K}$ 处表现出单一弛豫过程.

\section{2 结果与讨论}

\section{1 单晶到单晶转变}

化合物 $\mathbf{1} \cdot \mathrm{H}_{2} \mathrm{O}$ 到化合物 $\mathbf{1}$ 的单晶-单晶转变, 可以从 化合物 $\mathbf{1} \cdot \mathrm{H}_{2} \mathrm{O}$ 在 $100 \mathrm{~K}$ 和 $293 \mathrm{~K}$ 及 $\mathbf{1}$ 在 $173 \mathrm{~K}$ 和 $293 \mathrm{~K}$ 单晶结构、热重分析和其变温粉末 XRD 数据得到证实. 化合物的单晶结构信息如表 1 所示.

在 $293 \mathrm{~K}$ 时, 化合物 $\mathbf{1} \cdot \mathrm{H}_{2} \mathrm{O}$ 结晶于单斜晶系, 空间 群为 $P 2_{1} / c$, 其晶体结构如图 1a 所示. 晶体结构表明化 合物 $1 \cdot \mathrm{H}_{2} \mathrm{O}$ 由一维阴离子链 $\left[\mathrm{Fe}_{2}(\mathrm{Ox})_{2} \mathrm{Cl}_{4}\right]^{2-}$ 、二甲胺阳离 子和结晶水分子组成, 其中的 $\left[\mathrm{Fe}_{2}(\mathrm{Ox})_{2} \mathrm{Cl}_{4}\right]^{2-}$ 一维阴离 子链是由 $\mathrm{Fe}$ 原子通过 2 个桥连草酸根和 2 个位于端末 位的 $\mathrm{Cl}$ 原子链接形成. 该 $\left[\mathrm{Fe}_{2}(\mathrm{ox})_{2} \mathrm{Cl}_{4}\right]^{2-}$ 链是以连接 $\mathrm{Fe} 1$
和 $\mathrm{Fe} 2$ 原子的草酸基团为中心沿 $c$ 轴方向展开.

相邻 $\mathrm{Fe} 1$ 和 $\mathrm{Fe} 2$ 原子上一对氯原子(Cl1 和 $\mathrm{Cl}$ ) 处于 同向位置, 另一对氯原子 $(\mathrm{Cl} 2$ 和 $\mathrm{Cl} 3)$ 处于反向位置. 而 相邻铁原子(Fe1 和 Fe1a, Fe2 和 Fe2b; 对称代码: a: 2$x,-y,-z ; \mathrm{b}: 2-x,-y, 1-z)$ 上氯原子都是处于反向 位置, 类似的链结构在化合物 $\{[\mathrm{K}(18$-crown-6)]$\left.\left[\mathrm{Mn}\left(\mathrm{H}_{2} \mathrm{O}\right)_{2} \mathrm{Cr}(\mathrm{ox})_{3}\right]\right\} \quad$ (18-crown-6 $=\mathrm{C}_{12} \mathrm{H}_{24} \mathrm{O}_{6}, \quad$ ox $=$ oxalate)中也有报道 ${ }^{[26]}$. 相邻的两个一维链在沿 $a$ 轴方向 以相同的方式排列, 而沿 $b$ 轴方向是反向排列(图 S1). 二甲胺阳离子通过氢键 $\left(\mathrm{N} 2 \cdots \mathrm{Cl}_{4}^{4} 、 \mathrm{~N} 1 \cdots \mathrm{O} 1 、 \mathrm{~N} 1 \cdots \mathrm{Cl} 2\right.$ 、 $\left.\mathrm{N} 2^{\prime} \cdots \mathrm{Cl} 1^{3} 、 \mathrm{~N} 2^{\prime} \cdots \mathrm{Cl} 13 、 \mathrm{~N} 1^{\prime} \cdots \mathrm{Cl} 2^{6} 、 \mathrm{~N} 1^{\prime} \cdots \mathrm{O} 1\right)$ 或间接通过结 晶水 $\left(\mathrm{N} 2 \cdots \mathrm{O} 1 \mathrm{~W} \cdots \mathrm{O} 2 、 \mathrm{~N} 2 \cdots \mathrm{O} 1 \mathrm{~W} \cdots \mathrm{Cl1}{ }^{1} 、 \mathrm{~N} 2 \cdots \mathrm{O} 1 \mathrm{~W} \cdots \mathrm{Cl}^{2}{ }^{2}\right)$ 与一维阴离子链连接. 相邻的链通过与结晶水 $\left(\mathrm{O} 1 \mathrm{~W} \cdots \mathrm{O} 2 、 \mathrm{O} 1 \mathrm{~W} \cdots \mathrm{Cl1}^{1} 、 \mathrm{O} 1 \mathrm{~W} \cdots \mathrm{Cl}^{2} 、 \mathrm{O} 1 \mathrm{~W} \cdots \mathrm{N} 2\right)$ 和二 甲胺阳离子 $\left(\mathrm{N} 2 \cdots \mathrm{Cl} 4^{4} 、 \mathrm{~N} 1 \cdots \mathrm{O} 1 、 \mathrm{~N} 1 \cdots \mathrm{Cl} 2 、 \mathrm{~N} 2^{\prime} \cdots \mathrm{Cl}^{3}\right.$ 、 $\left.\mathrm{N} 2^{\prime} \cdots \mathrm{Cl} 3 、 \mathrm{~N} 1^{1} \cdots \mathrm{Cl} 2^{6} 、 \mathrm{~N} 1{ }^{\prime} \cdots \mathrm{O} 1\right)$ 间形成的氢键相互连接, 进而形成了二维和三维结构(对称代码: $1: 1-x,-y$, $-z ; 2:+x, 1 / 2-y,-1 / 2+z ; 3:+x, 1 / 2-y, 1 / 2+z ; 4$ : $1-x, 1 / 2+y, 1 / 2-z ; 6:+x,-1 / 2-y, 1 / 2+z$. 图 3、图 $\mathrm{S} 2$ 和表 $\mathrm{S} 1$ ).

热重分析表明，化合物 $\mathbf{1} \cdot \mathrm{H}_{2} \mathrm{O}$ 在 $352 \mathrm{~K}$ 之前保持稳 定, 在 $352 \mathrm{~K}$ 开始失重, 至 $463 \mathrm{~K}$ 失重大约 $3.3 \%$, 其失 去的部分为客体水(图 2). 当化合物 $\mathbf{1} \cdot \mathrm{H}_{2} \mathrm{O}$ 在真空条件 下加热至 $390 \mathrm{~K}$, 并保持 $2 \mathrm{~h}$, 晶体中的客体水分子可完 全被除去, 得到化合物 1 的单晶. 化合物在单晶到单晶 的转变过程中颜色并没有发生明显变化，但化合物的粉 末在失去水分子后会从黄色变成棕黄色, 如图 $2 \mathrm{a}$ 所示. 化合物 $\mathbf{1} \cdot \mathrm{H}_{2} \mathrm{O}$ 脱去客体水分子后转变成了化合物 1 . 这 一点从其在 $480 \mathrm{~K}$ 之前未出现任何失重以及其粉末 XRD 数据得到进一步证实. 此外, XRD 数据(图 S3)

表 1 化合物 $\mathbf{1} \cdot \mathrm{H}_{2} \mathrm{O}$ 和 1 在不同温度的单晶结构信息

Table 1 Crystallographic data of $\mathbf{1} \cdot \mathrm{H}_{2} \mathrm{O}$ and $\mathbf{1}$ at different temperatures

\begin{tabular}{|c|c|c|c|c|}
\hline & \multicolumn{2}{|c|}{$\mathbf{1} \cdot \mathrm{H}_{2} \mathrm{O}$} & \multicolumn{2}{|c|}{1} \\
\hline 温度/K & $100(2)$ & 293(2) & $173(2)$ & $293(2)$ \\
\hline 分子式 & $\mathrm{C}_{8} \mathrm{H}_{18} \mathrm{O}_{9} \mathrm{~N}_{2} \mathrm{Fe}_{2} \mathrm{Cl}_{4}$ & $\mathrm{C}_{8} \mathrm{H}_{18} \mathrm{O}_{9} \mathrm{~N}_{2} \mathrm{Fe}_{2} \mathrm{Cl}_{4}$ & $\mathrm{C}_{8} \mathrm{H}_{16} \mathrm{O}_{8} \mathrm{~N}_{2} \mathrm{Fe}_{2} \mathrm{Cl}_{4}$ & $\mathrm{C}_{8} \mathrm{H}_{16} \mathrm{O}_{8} \mathrm{~N}_{2} \mathrm{Fe}_{2} \mathrm{Cl}_{4}$ \\
\hline 相对分子质量 & 539.74 & 539.74 & 521.73 & 521.73 \\
\hline 晶体颜色 & 黄色 & 黄色 & 黄色 & 黄色 \\
\hline 晶系 & Monoclinic & Monoclinic & Monoclinic & Monoclinic \\
\hline 空间群 & $P 2_{1} / c$ & $P 2_{1} / c$ & $P 2_{1} / c$ & $P 2_{1} / c$ \\
\hline$b / \mathrm{nm}$ & $1.37526(6)$ & $1.40077(8)$ & $1.35867(3)$ & $1.37243(6)$ \\
\hline$c / \mathrm{nm}$ & $1.35486(6)$ & $1.35620(8)$ & $1.36300(3)$ & $1.36689(7)$ \\
\hline$\alpha /\left(^{\circ}\right)$ & 90 & 90 & 90 & 90 \\
\hline$\beta /\left(^{\circ}\right)$ & $107.116(5)$ & $107.474(6)$ & $108.379(3)$ & $108.439(6)$ \\
\hline$\gamma /\left(^{\circ}\right)$ & 90 & 90 & 90 & 90 \\
\hline$V / \mathrm{nm}^{3}$ & $1.92963(15)$ & $1.9849(2)$ & $1.90840(8)$ & $1.94316(17)$ \\
\hline$Z$ & 4 & 4 & 4 & 4 \\
\hline GOOFs & 1.074 & 1.092 & 1.006 & 1.028 \\
\hline$R_{1}[I>2 \sigma(I)]$ & 0.0380 & 0.0486 & 0.0320 & 0.0391 \\
\hline$w R_{2}$ (all data) & 0.1091 & 0.1336 & 0.0578 & 0.0964 \\
\hline
\end{tabular}

${ }^{a} R_{1}=\sum|| F_{o}|-| F_{c}\left|/ \sum\right| F_{o} \mid ;{ }^{b} w R_{2}=\left\{\sum\left[w\left(F_{o}{ }^{2}-F_{c}{ }^{2}{ }^{2}\right] / \sum\left[w\left(F_{o}{ }^{2}\right)^{2}\right]\right\}\right.$. 


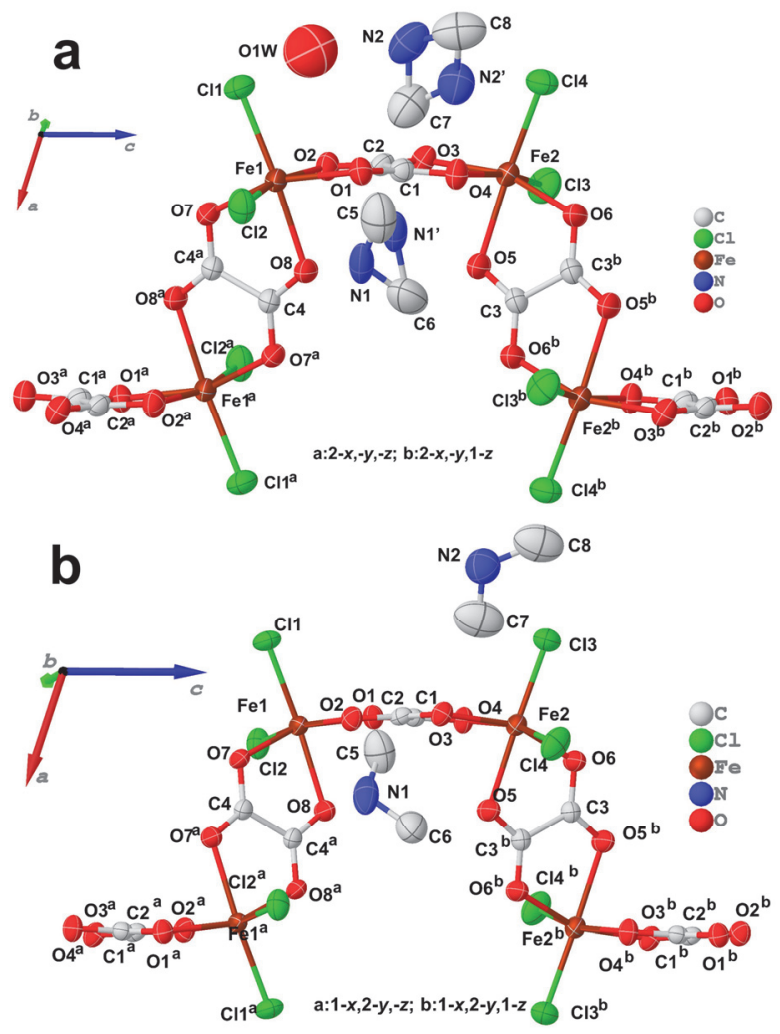

图 1 (a) 化合物 $\mathbf{1} \cdot \mathrm{H}_{2} \mathrm{O}$ 在 $293 \mathrm{~K}$ 的结构片段; (b) 化合物 $\mathbf{1}$ 在 $293 \mathrm{~K}$ 的结构片段. 为了图示清晰, 所有的氢原子都均被忽略

Figure 1 ORTEP drawing of a fragment of the chain motif in $\mathbf{1} \cdot \mathrm{H}_{2} \mathrm{O}$ (a) and 1 (b) at $293 \mathrm{~K}$. All $\mathrm{H}$ atoms are omitted for clarity

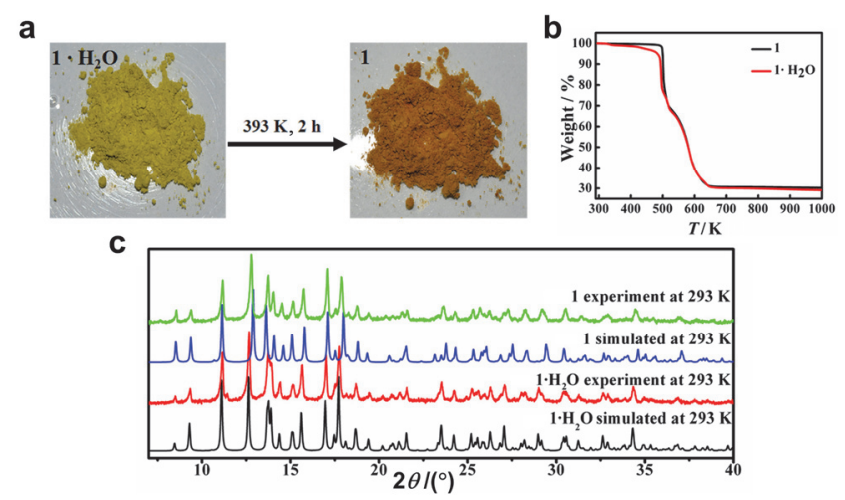

图 2 (a) $1 \cdot \mathrm{H}_{2} \mathrm{O}$ 到 $\mathbf{1}$ 的转换的粉末颜色变化; (b) $1 \cdot \mathrm{H}_{2} \mathrm{O}$ 和 $\mathbf{1}$ 的热重数 据; (c) $1 \cdot \mathrm{H}_{2} \mathrm{O}$ 和 $\mathbf{1}$ 的粉末 XRD 数据

Figure 2 (a) The color change in powder of $\mathbf{1} \cdot \mathrm{H}_{2} \mathrm{O}$ transfer to $\mathbf{1}$. (b) TG of $\mathbf{1} \cdot \mathrm{H}_{2} \mathrm{O}$ and $\mathbf{1}$. (c) PXRD of $\mathbf{1} \cdot \mathrm{H}_{2} \mathrm{O}$ and $\mathbf{1}$

还证明化合物 1 在空气气氛中放置 $4 \mathrm{~h}$ 后可转变成化合 物 $1 \cdot \mathrm{H}_{2} \mathrm{O}$, 表明该单晶-单晶转变过程是可逆的.

化合物 1 在 $293 \mathrm{~K}$ 的单晶结构测定表明其基本构架 和化合物 $\mathbf{1} \cdot \mathrm{H}_{2} \mathrm{O}$ 相同. 如图 $1 \mathrm{~b}$ 所示, 化合物 $\mathbf{1}$ 同样结晶 于单斜晶系, 空间群为 $P 2_{1} / c$. 不同的是二甲胺阳离子与 一维阴离子链 $\left(\mathrm{N} 2 \cdots \mathrm{Cl}^{2} 、 \mathrm{~N} 2 \cdots \mathrm{Cl} 4^{3} 、 \mathrm{~N} 1 \cdots \mathrm{O}^{1} 、 \mathrm{~N} 1 \cdots \mathrm{Cl} 2 、\right.$ $\mathrm{N} 1 \cdots \mathrm{O} 1)$ 和相邻一维阴离子链间 $\left(\mathrm{N} 2 \cdots \mathrm{Cl}^{2} 、 \mathrm{~N} 2 \cdots \mathrm{Cl} 4^{3}\right.$ 、
$\left.\mathrm{N} 1 \cdots \mathrm{O} 5^{1}\right)$ 相互连接的氢键种类和强度发生了变化 (对称 代码: 1: $-1-x, 1 / 2+y,-3 / 2-z ; 2:-2-x,-y,-2$ $-z ; 3:-2-x, 1 / 2+y,-3 / 2-z$; 图 3、图 S4 和表 S1). 因为客体水的缺失, 化合物 $\mathbf{1}$ 的 $a$ 轴和 $b$ 轴均较化合物 $1 \cdot \mathrm{H}_{2} \mathrm{O}$ 的 $a$ 轴和 $b$ 轴短. 为了适应 $a$ 轴和 $b$ 轴的收缩, 化 合物 $\mathbf{1}$ 的 $c$ 轴稍有增长, 从 $1.35620 \mathrm{~nm}\left(\mathbf{1} \cdot \mathrm{H}_{2} \mathrm{O}\right)$ 增长至 $1.36689 \mathrm{~nm}$ (1), 进而导致化合物 $\mathbf{1}$ 单胞体积 (1.94316(17) $\mathrm{nm}^{3}$ )略小于 $\mathbf{1} \cdot \mathrm{H}_{2} \mathrm{O}$ 的单胞体积(1.9849(2) $\mathrm{nm}^{3}$ ). 同时, 化合物 $\mathbf{1} \cdot \mathrm{H}_{2} \mathrm{O}$ 沿着 $c$ 轴方向的与草酸配位 的两个相邻铁离子之间的距离 $(\mathrm{Fe} 1 \mathrm{a}-\mathrm{Fe} 10.5483 \mathrm{~nm}$, $\mathrm{Fe} 1-\mathrm{Fe} 20.5429 \mathrm{~nm}, \mathrm{Fe} 2-\mathrm{Fe} 2 \mathrm{~b} 0.5507 \mathrm{~nm}$, 对称代码: a: $2-x,-y,-z ; \mathrm{b}: 2-x,-y, 1-z)$ 比化合物 $\mathbf{1}$ 略有减小 (Fe1a-Fe1 0.5509 nm, Fe1-Fe2 0.5434 nm, Fe2-Fe2b $0.5530 \mathrm{~nm}$, 对称代码: $\mathrm{a}:-1-x,-y,-2-z ; \mathrm{b}:-1$ $-x,-y,-1-z$; 图 S5). 此外, 在化合物 $\mathbf{1} \cdot \mathrm{H}_{2} \mathrm{O}$ 中的 两个二甲胺阳离子都处于二重无序状态. 随着客体水分 子的脱去, 化合物 $\mathbf{1}$ 中两个二甲胺阳离子都处于有序状 态(图 $1 b$ 和图 $3 b)$.

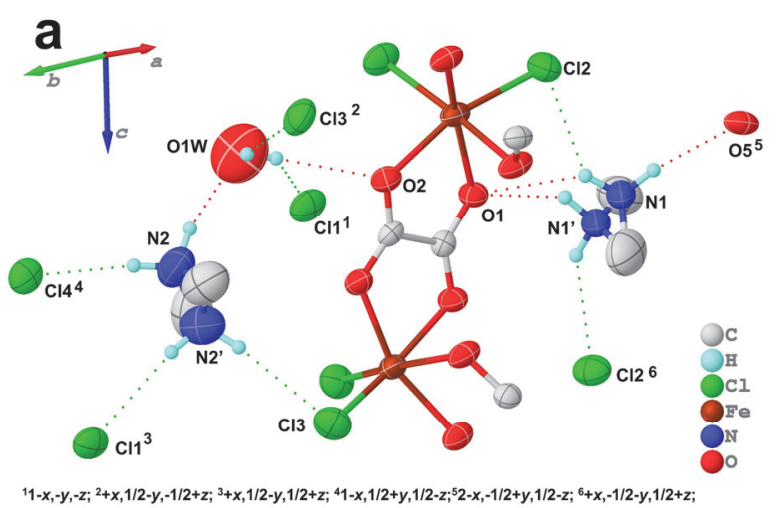

$11-x,-y,-z ;{ }^{2}+x, 1 / 2-y,-1 / 2+z ;{ }^{3}+x, 1 / 2-y, 1 / 2+z ;{ }^{41-x}, 1 / 2+y, 1 / 2-z ; 52-x,-1 / 2+y, 1 / 2-z ;{ }^{6}+x,-1 / 2-y, 1 / 2+z ;$
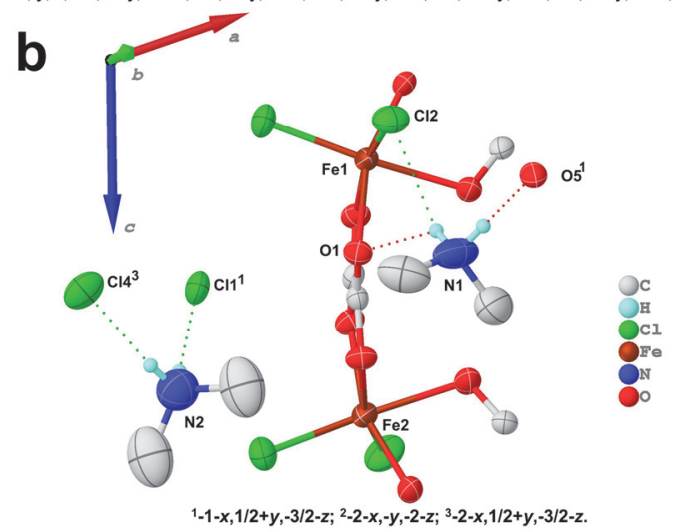

图 3 化合物 $1 \cdot \mathrm{H}_{2} \mathrm{O}(\mathrm{a})$ 和 $\mathbf{1}(\mathrm{b})$ 在 $293 \mathrm{~K}$ 的不对称单元图. 为了图示清 晰, 所有非氢键的氢原子均被忽略

Figure 3 The asymmetric unit of $\mathbf{1} \cdot \mathrm{H}_{2} \mathrm{O}$ (a) and $\mathbf{1}$ (b) at $293 \mathrm{~K}$, respectively. The non-hydrogen bonded $\mathrm{H}$ atoms are omitted for clarity

\section{2 介电性质}

\subsection{1 介电性能}

化合物 $1 \cdot \mathrm{H}_{2} \mathrm{O}$ 在不同温度 $(6 \mathrm{~K}$ 到 $300 \mathrm{~K})$ 和不同频率 $\left(10^{2.5} \mathrm{~Hz}\right.$ 到 $\left.10^{5.0} \mathrm{~Hz}\right)$ 下的介电数据 $\left(\varepsilon=\varepsilon^{\prime}-\mathrm{i} \varepsilon^{\prime \prime}\right)$ 如图 4 和 
图 S6 所示. 在 $6 \mathrm{~K}$ 至 $40 \mathrm{~K}$ 之间, 化合物 $1 \cdot \mathrm{H}_{2} \mathrm{O}$ 的介电 常数几乎保持不变, 介电常数约为 6.1. 随着温度的升 高, 在 $60 \mathrm{~K}$ 附近介电常数出现了一个阶梯状的介电峰, 介电常数从 6.1 增加到了 6.8. 该介电峰随着频率的升高 而向高温区移动, 且峰值随频率的增加而减小. 随着温 度的继续增加, 在 $250 \mathrm{~K}$ 附近出现了一个台阶状的介电 峰, 介电常数从 8.5 增加到了 13.0. 两个介电峰随着频 率的升高向高温区移动, 介电峰值随频率的增加而减 小, 表现出显著的频率依赖性. 从介电损耗和介电常数 虚部的数据也可以看出上述介电异常 (图 $4 \mathrm{~b} \sim 4 \mathrm{~d}$ ). 为了 更加清楚地区分三个弛豫行为, 对其分别命名为 $\alpha-、 \beta-$ 、 $\gamma$-弛豫. 由图 $4 \mathrm{~b}$ 的介电损耗可知, $250 \mathrm{~K}$ 附近介电损耗 峰呈现出了明显的弛豫的特点 ( $\alpha$-弛豫), 从 $10^{2.5} \mathrm{~Hz}$ 到 $10^{5.0} \mathrm{~Hz}$, 随着频率增加介电损耗峰变小, 并且逐步向高 温移动, 数值从 $0.136\left(250 \mathrm{~K}, 10^{2.5} \mathrm{~Hz}\right)$ 移至 $0.120(300$ $\mathrm{K}, 10^{5.0} \mathrm{~Hz}$ ). 值得注意的是, $60 \mathrm{~K}$ 的介电损耗和虚部数 据表明该介电峰是由两个弛豫过程组成, 一个在 $80 \mathrm{~K}$ 附近的肩峰( $\beta$-弛豫)和一个在 $50 \mathrm{~K}$ 附近的凸峰( $\gamma$-弛豫). $\beta$-弛豫和 $\gamma$-弛豫的介电损耗峰也具有和 $\alpha$-弛豫介电损耗 峰相同的温度和频率依赖关系. $\gamma$-弛豫损耗峰随频率从 $10^{2.5} \mathrm{~Hz}$ 升高到 $10^{5.0} \mathrm{~Hz}$, 峰值对应的温度从 $60 \mathrm{~K}$ 转移到 $100 \mathrm{~K}$ 附近(图 4b). 同样通过介电虚部也可以清楚地区 分出 $\alpha$-弛豫, $\beta$-弛豫和 $\gamma$-弛豫三个弛豫过程(图 4c, $4 \mathrm{~d}$ ).

与化合物 $\mathbf{1} \cdot \mathrm{H}_{2} \mathrm{O}$ 不同, 化合物 $\mathbf{1}$ 表现出不同的介电
行为. 如图 5 所示, 在 $6 \mathrm{~K}$ 至 $40 \mathrm{~K}$ 之间，化合物 1 的介 电常数随温度升高缓慢升高, 在 $40 \mathrm{~K}$ 时介电常数约为 6.4. 随着温度的进一步升高, 在 $80 \mathrm{~K}$ 附近出现了一个 阶梯状的介电峰, 介电常数从 6.4 增加到了 7.0. 同时, 介电峰也随着频率的升高而向高温区移动, 介电常数的 数值随频率的增加而减小, 并且随着频率的增加而逐渐 减小. 该介电弛豫的温区与化合物 $\mathbf{1} \cdot \mathrm{H}_{2} \mathrm{O}$ 中 $\beta$-弛豫温区 相同, 但比 $\mathbf{1} \cdot \mathrm{H}_{2} \mathrm{O}$ 的弛豫温区要更宽. 因此, 我们称该 弛豫也为 $\beta$-弛豫. 同样, 从介电损耗和介电虚部的数据 也可以看出上述介电异常(图 5b, 5c).

由图 $5 \mathrm{~b}$ 的介电损耗可知, $80 \mathrm{~K}$ 附近介电损耗峰呈 现出了明显的弛豫的特点( $\beta$-弛豫), 从 $10^{2.5} \mathrm{~Hz}$ 到 $10^{5.0}$ $\mathrm{Hz}$ ，随着频率增加介电损耗峰变大，并且逐步向高温移 动. 其数值从 $0.0502\left(76 \mathrm{~K}, 10^{3.0} \mathrm{~Hz}\right)$ 移至 $0.00765(98 \mathrm{~K}$, $10^{5.0} \mathrm{~Hz}$ ). 由介电虚部图 $5 \mathrm{c}$ 可知, 介电常数虚部有相似 的频率依赖性.

\subsection{2 介电弛豫}

很明显, $1 \cdot \mathrm{H}_{2} \mathrm{O}$ 和 1 中频率和温度依赖的介电变化 符合 Debye 型介电弛豫行为, 该类型介电弛豫行为很有 可能跟化合物中的二甲胺阳离子和客体水分子的动态 变化有关. 为了进一步揭示介电弛豫的结构来源, 基于 阿伦尼乌斯公式，对介电虚部进行拟合，分别计算出化 合物 $1 \cdot \mathrm{H}_{2} \mathrm{O}$ 和 1 的弛豫过程的活化能和弛豫时间.
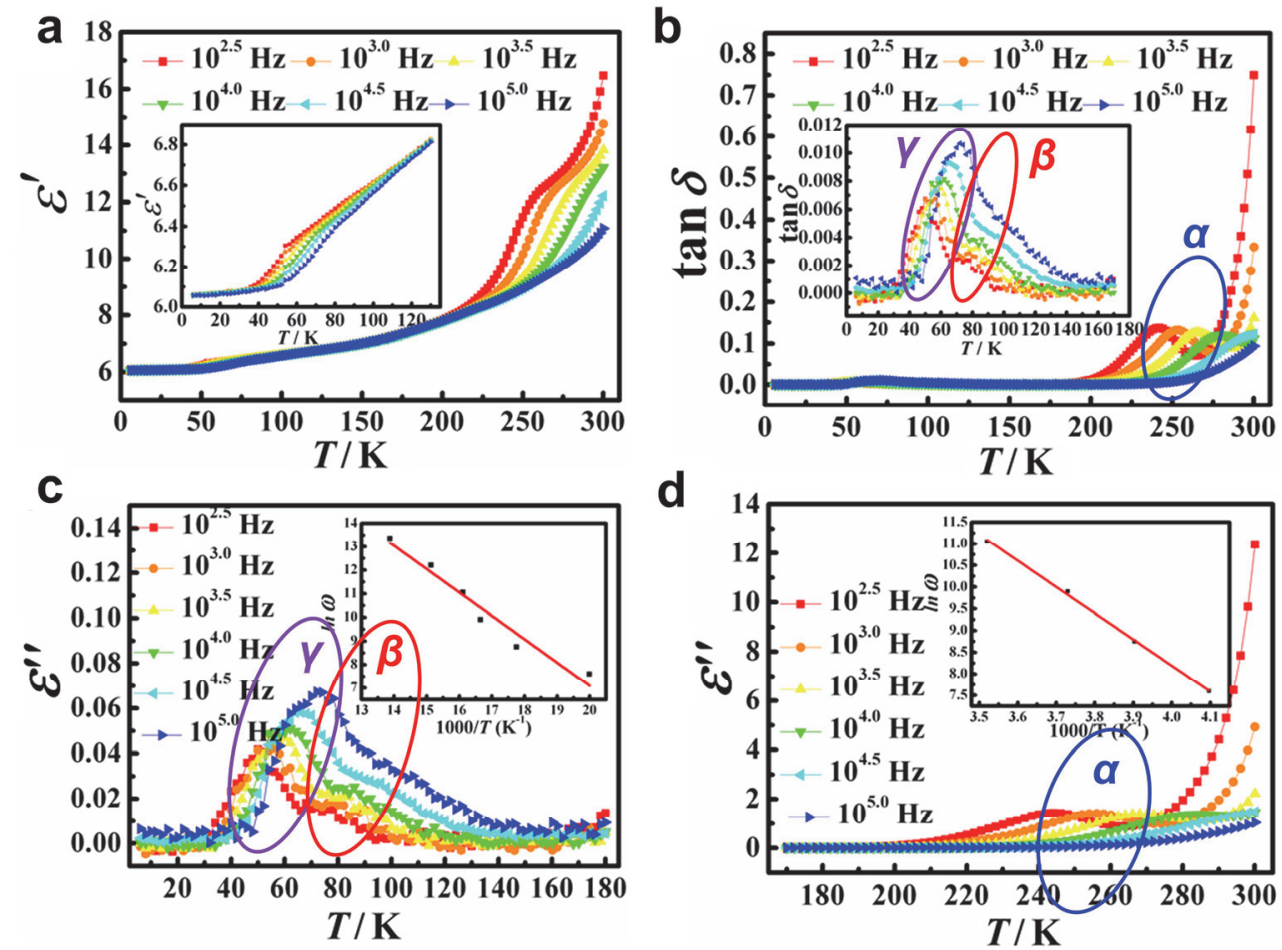

图 $41 \cdot \mathrm{H}_{2} \mathrm{O}$ 的变温介电温谱图. (a) 不同频率下的介电实部图, 内插图为低温区介电实部图; (b) 不同频率下的介电损耗图; 在 $50 \mathrm{~K}$ (c), $250 \mathrm{~K}(\mathrm{~d})$ 附近, 不同频率下的介电虚部图, 内插图为基于阿伦尼乌斯公式的拟合图.

Figure 4 Variable-temperature permittivity spectrum of $1 \cdot \mathrm{H}_{2} \mathrm{O}$. The real part $\left(\varepsilon^{\prime}\right)$ of the dielectric constant (a) and dielectric loss (b) dependence on temperature at different frequency for $\mathbf{1} \cdot \mathrm{H}_{2} \mathrm{O}$, inset is the real part $\left(\varepsilon^{\prime}\right)$ of the dielectric constant (a) and dielectric loss (b) at low temperature. The imaginary parts of dielectric constant dependence on different frequency near $50 \mathrm{~K}$ (c) and $250 \mathrm{~K}$ (d) of $1 \cdot \mathrm{H}_{2} \mathrm{O}$, inset is the fitting diagram of Arrhenius formula. 

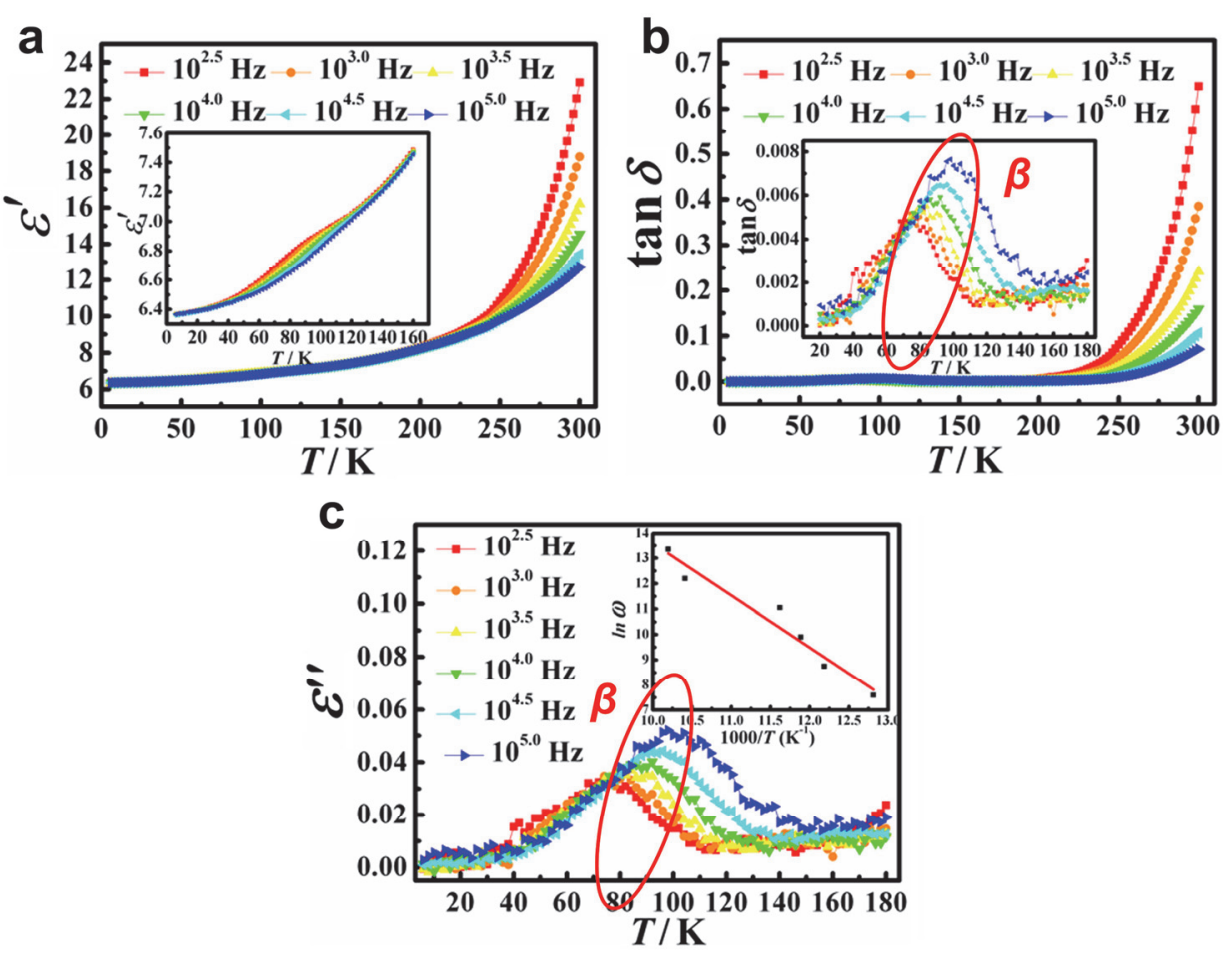

图 5 化合物 $\mathbf{1}$ 的变温介电温谱图. (a) 不同频率下的介电实部图, 内插图为低温区介电实部图; (b) 不同频率下的介电损耗图, 内插图为 $80 \mathrm{~K}$ 附 近的介电损耗图; (c) 不同频率下的介电虚部图, 内插图为基于阿伦尼乌斯公式的拟合图.

Figure 5 Variable-temperature permittivity spectrum of $\mathbf{1}$. The real part $\left(\varepsilon^{\prime}\right)$ of the dielectric constant (a) and dielectric loss (b) dependence on temperature at different frequency for $\mathbf{1}$, inset is the dielectric loss at around $80 \mathrm{~K}$. (c) The imaginary parts of dielectric constant dependence on different frequency, inset is the fitting diagram of Arrhenius formula.

根据 Arrhenius 公式,

$$
\frac{1}{\tau}=\omega_{0} e^{-\frac{E_{\mathrm{a}}}{k_{\mathrm{B}} T}}
$$

其中 $E_{\mathrm{a}}$ 是活化能, $\tau$ 是驰豫时间, $\omega_{0}$ 是指前因子 $\left(\omega_{0}=\right.$ $\left.1 / \tau_{0}\right), k_{\mathrm{B}}$ 是玻尔兹曼常数. 对三个介电弛豫过程进行计 算可以得到相应的 $E_{\mathrm{a}}$ 和 $\tau_{0}$ (表 2).

表 2 化合物 $\mathbf{1} \cdot \mathrm{H}_{2} \mathrm{O}$ 和 $\mathbf{1}$ 在弛豫过程中的 $E_{\mathrm{a}}$ 和 $\tau_{0}$

Table 2 Activation energy $\left(E_{\mathrm{a}}\right)$ and relaxation time $\left(\tau_{0}\right)$ for each relaxation process in $\mathbf{1} \cdot \mathrm{H}_{2} \mathrm{O}$ and $\mathbf{1}$

\begin{tabular}{cccccc}
\hline \multirow{2}{*}{ 弛豫 } & \multicolumn{2}{c}{$\mathbf{1} \cdot \mathrm{H}_{2} \mathrm{O}$} & & \multicolumn{2}{c}{$\mathbf{1}$} \\
\cline { 2 - 3 } \cline { 5 - 6 } & $E_{\mathrm{a}}$ & $\tau_{0}$ & & $E_{\mathrm{a}}$ & $\tau_{0}$ \\
\hline$\alpha$-弛豫 & $0.521 \mathrm{eV}$ & $9.0 \times 10^{-15} \mathrm{~s}$ & & \\
$\beta$-弛豫 & $0.148 \mathrm{eV}$ & $5.88 \times 10^{-14} \mathrm{~s}$ & & $0.1768 \mathrm{eV}$ & $1.58 \times 10^{-15} \mathrm{~s}$ \\
$\gamma$-弛豫 & $0.0855 \mathrm{eV}$ & $2.07 \times 10^{-12} \mathrm{~s}$ & & \\
\hline
\end{tabular}

化合物 $1 \cdot \mathrm{H}_{2} \mathrm{O}$ 中 $\alpha$-弛豫过程的活化能 $E_{\mathrm{a}}$ 为 $0.521 \mathrm{eV}$, 弛豫时间 $\tau_{0}$ 为 $9.0 \times 10^{-15} \mathrm{~s}$. 对比 $1 \cdot \mathrm{H}_{2} \mathrm{O}$ 在 $293 \mathrm{~K}$ 和 $100 \mathrm{~K}$ 的结构可以看出, 在该温区, 其中的一个 $\mathrm{Me}_{2} \mathrm{NH}_{2}{ }^{+}$阳离 子经历了从无序到有序的过程(图 S7). 因此, 我们认定 $1 \cdot \mathrm{H}_{2} \mathrm{O}$ 中 $\alpha$-弛豫过程主要归因于 $\mathrm{Me}_{2} \mathrm{NH}_{2}{ }^{+}$阳离子的有 序-无序运动. 同时, $\alpha$-弛豫的活化能大小也和很多 文献报道的化合物，如 $\left[\left(\mathrm{CH}_{3}\right)_{2} \mathrm{NH}_{2}\right]\left[\mathrm{Zn}(\mathrm{HCOO})_{3}\right]$ 、 $\left[\left(\mathrm{CH}_{3}\right)_{2} \mathrm{NH}_{2}\right]\left[\mathrm{M}(\mathrm{HCOO})_{3}\right](\mathrm{M}=\mathrm{Ni}, \mathrm{Mn})$ 中的二甲胺阳离
子有序-无序运动所需要的活化能大小相一致 ${ }^{[27-28]}$. 由 于化合物 $\mathbf{1}$ 中的两个 $\mathrm{Me}_{2} \mathrm{NH}_{2}{ }^{+}$阳离子在整个温区都为有 序的状态, 因此在 1 中没有表现出 $\alpha$-弛豫.

化合物 $\mathbf{1} \cdot \mathrm{H}_{2} \mathrm{O}$ 和 $\mathbf{1}$ 中的 $\beta$-弛豫, 不仅其温度区间相 近, 活化能也都十分的接近. 结合其相似的结构, 我们 可以判断该弛豫过程应该有着相同的起源. 对比二者的 结构, 我们推断认为该弛豫与二甲胺阳离子与阴离子一 维链间的氢键他豫有关. 但是 $1 \cdot \mathrm{H}_{2} \mathrm{O}$ 中的弛豫时间明显 长于 $\mathbf{1}$ 的弛豫时间. 这可能跟氢键的强度发生变化有关. 对比 $293 \mathrm{~K}$ 化合物 $\mathbf{1} \cdot \mathrm{H}_{2} \mathrm{O}$ 和 $\mathbf{1}$ 中的氢键发现(化合物

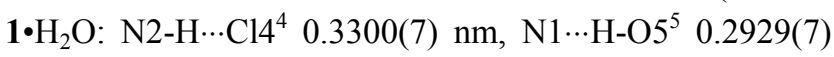

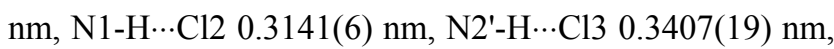
$\mathrm{N} 1{ }^{\prime}-\mathrm{H} \cdots \mathrm{O} 1 \quad 0.2778(14) \mathrm{nm}, \mathrm{N} 1-\mathrm{H} \cdots \mathrm{O} 1 \quad 0.3095(6) \mathrm{nm}$, $\mathrm{N} 2{ }^{\prime}-\mathrm{H}^{\prime} \cdots \mathrm{Cl} 1^{3} 0.3422(19) \mathrm{nm}, \mathrm{N} 1{ }^{\prime}-\mathrm{H} 1 \cdots \mathrm{Cl} 2^{6} 0.3344(15) \mathrm{nm}$, 对称代码: $3:+x, 1 / 2-y, 1 / 2+z ; 4: 1-x, 1 / 2+y, 1 / 2-z$; $5: 2-x,-1 / 2+y, 1 / 2-z ; 6:+x,-1 / 2-y, 1 / 2+z$. 化

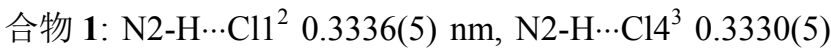
$\mathrm{nm}, \mathrm{N} 1-\mathrm{H} \cdots \mathrm{Cl} 20.3189(4) \mathrm{nm}, \mathrm{N} 1-\mathrm{H} \cdots \mathrm{O}^{1}{ }^{1} 0.2920(5) \mathrm{nm}$, $\mathrm{N} 1-\mathrm{H} \cdots \mathrm{O} 10.3034(5) \mathrm{nm}$; 对称代码: 1: $-1-x, 1 / 2+y$, $-3 / 2-z ; 2:-2-x,-y,-2-z ; 3:-2-x, 1 / 2+y$, $-3 / 2-z$ ), 从氢键数量和强度上看, 化合物 $1 \cdot \mathrm{H}_{2} \mathrm{O}$ 中的 氢键明显多和强于化合物 1 中的氢键. 氢键强度更大, 氢键作用更强, 使得诱发化合物 $\mathbf{1} \cdot \mathrm{H}_{2} \mathrm{O}$ 发生弛豫需要更 长的时间. 这与通过计算拟合得到的化合物 $1 \cdot \mathrm{H}_{2} \mathrm{O}$ 的弛 
豫时间 $\left(5.88 \times 10^{-14} \mathrm{~s}\right)$ 比化合物 $\mathbf{1}$ 的枵豫时间 $(1.58 \times$ $\left.10^{-15} \mathrm{~s}\right)$ 长的结果相吻合. 同时化合物 $\mathbf{1} \cdot \mathrm{H}_{2} \mathrm{O}$ 的活化能 $(0.148 \mathrm{eV})$ 比化合物 $\mathbf{1}$ 的活化能 $(0.1768 \mathrm{eV})$ 低可能是由 于化合物 $1 \cdot \mathrm{H}_{2} \mathrm{O}$ 脱去客体水之后, 导致与一维链有氢键 作用的二甲胺阳离子的周围环境改变, 影响了弛豫行 为.

而 $\gamma$-弛豫也仅仅存在于化合物 $\mathbf{1} \cdot \mathrm{H}_{2} \mathrm{O}$ 中, 活化能 $E_{\mathrm{a}}$ 为 $0.0855 \mathrm{eV}$, 弛豫时间 $\tau_{0}$ 为 $2.07 \times 10^{-12} \mathrm{~s}$. 很明显此弛 豫过程活化能小, 弛豫时间相对较长. 分析 $\mathbf{1} \cdot \mathrm{H}_{2} \mathrm{O}$ 在 $100 \mathrm{~K}$ 的晶体结构, 可以发现其中仍有一个 $\mathrm{Me}_{2} \mathrm{NH}_{2}{ }^{+}$阳 离子处于无序状态, 而且该弛豫的活化能与化合物 $\left(\mathrm{NH}_{4}\right)_{2}(\mathrm{adp})\left[\mathrm{Zn}_{2}(\mathrm{ox})_{3}\right] \cdot 3 \mathrm{H}_{2} \mathrm{O}(\mathrm{adp}=$ adipic acid 中由胺离 子的运动导致的弛豫的活化能十分接近 ${ }^{[29]}$. 因此, 可以 确定 $\gamma$-弛豫过程是客体水结合的无序 $\mathrm{Me}_{2} \mathrm{NH}_{2}{ }^{+}$阳离子 的运动造成的.

\section{3 磁性分析}

根据 $\mathbf{1} \cdot \mathrm{H}_{2} \mathrm{O}$ 和 $\mathbf{1}$ 在 $293 \mathrm{~K}$ 的单晶结构可知, 随着水 分子的失去, 二者 $\mathrm{Fe}-\mathrm{Fe}$ 之间的距离发生相应的变化, $\mathbf{1} \cdot \mathrm{H}_{2} \mathrm{O}$ 中的相邻两个铁离子之间的距离 (Fela-Fe1 $0.5483 \mathrm{~nm}, \mathrm{Fe} 1-\mathrm{Fe} 20.5429 \mathrm{~nm}, \mathrm{Fe} 2-\mathrm{Fe} 2 \mathrm{~b} 0.5507 \mathrm{~nm}$, 对 称代码: $\mathrm{a}: 2-x,-y,-z ; \mathrm{b}: 2-x,-y, 1-z$ ) 短于化合 物 1 中的相邻两个铁离子之间的距离( $\mathrm{Fe} 1 \mathrm{a}-\mathrm{Fe} 10.5509$ nm, Fe1-Fe2 0.5434 nm, Fe2-Fe2b $0.5530 \mathrm{~nm}$, 对称代码: $\mathrm{a}:-1-x,-y,-2-z ; \mathrm{b}:-1-x,-y,-1-z)$, 其磁 性可能会因此被影响(图 S5). 尽管化合物 $\mathbf{1} \cdot \mathrm{H}_{2} \mathrm{O}$ 的磁性 已经报道过 ${ }^{[30]}$, 但为了对比二者的磁性, 对 $\mathbf{1} \cdot \mathrm{H}_{2} \mathrm{O}$ 和 $\mathbf{1}$ 的磁性均进行了测试. 在 $1000 \mathrm{Oe}$ 的外加磁场下, 二者 在 $2 \mathrm{~K}$ 到 $300 \mathrm{~K}$ 温区内的磁化率如图 6 所示, 对于 $1 \cdot \mathrm{H}_{2} \mathrm{O}$, 其室温 $\chi_{\mathrm{m}} T$ 值为 $6.86 \mathrm{~cm}^{3} \cdot \mathrm{mol}^{-1} \cdot \mathrm{K}$ 并随着温度的 降低逐渐减小, 在 $2 \mathrm{~K}$ 降低至 $0.178 \mathrm{~cm}^{3} \cdot \mathrm{mol}^{-1} \cdot \mathrm{K}$, 表明 存在着反铁磁作用. 对 $100 \mathrm{~K}$ 到 $300 \mathrm{~K}$ 的磁化率数据进 行居里外斯定律进行拟合, 得到居里常数 $C=9.02$ $\mathrm{cm}^{3} \cdot \mathrm{mol}^{-1} \cdot \mathrm{K}$, 外斯常数 $\theta=-90.60 \mathrm{~K}$, 说明 $\mathbf{1} \cdot \mathrm{H}_{2} \mathrm{O}$ 中存 在反铁磁行为.

化合物 $\mathbf{1}$ 的磁性行为和化合物 $\mathbf{1} \cdot \mathrm{H}_{2} \mathrm{O}$ 基本相同, 其 室温 $\chi_{\mathrm{m}} T$ 值为 $6.88 \mathrm{~cm}^{3} \cdot \mathrm{mol}^{-1} \cdot \mathrm{K}$ 并随着温度的降低逐渐 减小, 在 $2 \mathrm{~K}$ 降低至 $0.078 \mathrm{~cm}^{3} \cdot \mathrm{mol}^{-1} \cdot \mathrm{K}$. 对 $100 \mathrm{~K}$ 到 300 $\mathrm{K}$ 的磁化率数据进行拟合, 得到居里常数 $C=9.55$ $\mathrm{cm}^{3} \cdot \mathrm{mol}^{-1} \cdot \mathrm{K}$, 外斯常数 $\theta=-111.57 \mathrm{~K}$. 为了阐明化合 物 $1 \cdot \mathrm{H}_{2} \mathrm{O}$ 和 1 的磁性离子之间的磁交换作用, 我们利用 基于经典一维链哈密顿算符的公式(2)对二者的磁性数 据进行了拟合 ${ }^{[31-32]}$. 根据进行计算, 对 $\mathbf{1} \cdot \mathrm{H}_{2} \mathrm{O}, J=$ $-8.585 \mathrm{~cm}^{-1}, g=2.0, R=3.85 \times 10^{-5}$; 而对化合物 $\mathbf{1}, J$ $=-8.496 \mathrm{~cm}^{-1}, g=2.0, R=4.788 \times 10^{-5}$. 其中 $J$ 是磁电 耦合系数, $g$ 是朗德因子, $R$ 是误差 $\left(R=\sum\left[\left(\chi_{\mathrm{m}} T\right)_{\mathrm{obs}}-\right.\right.$ $\left.\left.\left(\chi_{\mathrm{m}} T\right)_{\text {calcd }}\right]^{2} / \Sigma\left[\left(\chi_{\mathrm{m}} T\right)_{\mathrm{obs}}\right]^{2}\right)$.
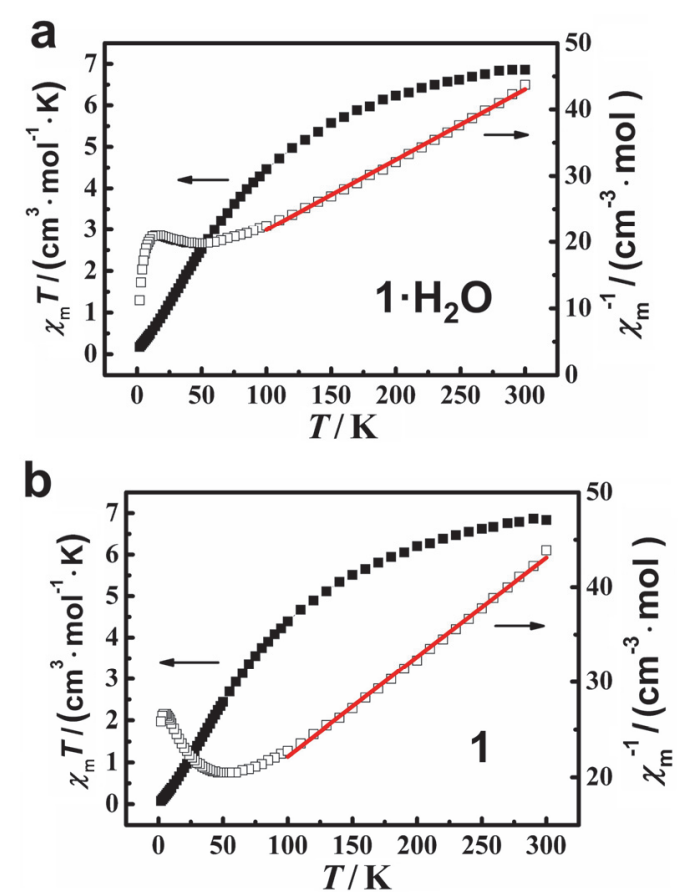

图 6 (a) $1 \cdot \mathrm{H}_{2} \mathrm{O}$ 的变温磁化率测试和(b) $\mathbf{1}$ 的变温磁化率测试 Figure 6 Plots of $\chi_{\mathrm{m}} T$ and $\chi_{\mathrm{m}}{ }^{-1}$ versus $T$ for $\mathbf{1} \cdot \mathrm{H}_{2} \mathrm{O}$ (a) and $\mathbf{1}$ (b)

$$
H=-J \sum_{i=1}^{N} S_{i} S_{i+1}
$$

通过对比两个化合物的 $J$ 值和外斯常数 $\theta$ 值可以看 出，尽管水分子的脱去会改变相邻两个铁离子之间的距 离, 但是化合物 $\mathbf{1}$ 和 $\mathbf{1} \cdot \mathrm{H}_{2} \mathrm{O}$ 的反铁磁相互作用没有明显 差别, 说明单晶到单晶转变中客体水分子的存在对 $\mathrm{Fe}-\mathrm{Fe}$ 之间的反铁磁耦合作用没有明显影响.

\section{3 结论}

本工作中，我们报道了化合物 $\left\{\left(\mathrm{Me}_{2} \mathrm{NH}_{2}\right)_{2}-\right.$ $\left.\left[\mathrm{Fe}_{2}(\mathrm{ox})_{2} \mathrm{Cl}_{4}\right] \cdot \mathrm{H}_{2} \mathrm{O}\right\}_{n} \quad(\mathrm{ox}=$ oxalate $)\left(\mathbf{1} \cdot \mathrm{H}_{2} \mathrm{O}\right)$ 和化合物 $\left\{\left(\mathrm{Me}_{2} \mathrm{NH}_{2}\right)_{2}\left[\mathrm{Fe}_{2}(\mathrm{ox})_{2} \mathrm{Cl}_{4}\right]\right\}_{n}$ (1) 之间的单晶到单晶转变, 并对二者的磁性及变温介电谱进行了研究. 研究结果表 明: 客体水分子的存在与否对化合物的磁性没有明显影 响, 但客体水分子的存在明显影响了化合物中客体胺和 氢原子的动态驰豫过程. 因此, 该研究工作为研究由客 体水分子引起的化合物结构变化及介电性质变化提供 了参考.

\section{4 实验部分}

\section{1 化合物合成}

化合物 $\mathbf{1} \cdot \mathrm{H}_{2} \mathrm{O}$ 是根据文献[30]报道方法进行制备的. 将 $5 \mathrm{mmol}$ 的 $\mathrm{FeCl}_{3} 、 5 \mathrm{mmol}$ 的草酸和 $5 \mathrm{mmol}$ 的氯化二 甲基胺溶解在 $10 \mathrm{~mL}$ 去离子水中. 室温挥发后即可得到 黄色块状晶体，产率 $45 \%$ (基于 $\mathrm{FeCl}_{3}$ ). 将 $\mathbf{1} \cdot \mathrm{H}_{2} \mathrm{O}$ 在真空 
条件下加热至 $390 \mathrm{~K}$ 保持 $2 \mathrm{~h}$ 得到化合物 1 .

化合物 1. $\mathrm{H}_{2} \mathrm{O}$ 元素分析理论值为 $\mathrm{C}: 17.80, \mathrm{H}: 3.36$, $\mathrm{N}: 5.12$; 实测值为 $\mathrm{C}: 17.85, \mathrm{H}: 3.18, \mathrm{~N}: 5.11 .1 \cdot \mathrm{H}_{2} \mathrm{O}$ 红外 数据 $\left(\mathrm{KBr}, \mathrm{cm}^{-1}\right): 3592.9(\mathrm{w}), 3444.4(\mathrm{w}), 3178.3(\mathrm{~m})$, $2800.3(\mathrm{w}), 2437.7$ (w), 1693.3 (s), 1610.3 (s), 1461.9 (m), $1346.1(\mathrm{~m}), 1301.8(\mathrm{~m}), 1012.5(\mathrm{~m}), 800.4(\mathrm{~m}), 474.4(\mathrm{~s})$, 445.5 (w) (图 S6).

化合物 1 元素分析理论值为 C: $18.42, \mathrm{H}: 3.09, \mathrm{~N}$ : 5.37; 实测值为 $\mathrm{C}: 18.43, \mathrm{H}: 3.34, \mathrm{~N}: 5.26 .1$ 红外数据 $\left(\mathrm{KBr}, \mathrm{cm}^{-1}\right)$ : $3589.1(\mathrm{w}), 3415.5(\mathrm{w}), 3158.9(\mathrm{~m}), 2802.2$ (w), 2435.8 (w), 1695.2 (s), 1606.5 (s), 1461.9 (m), 1346.1 (m), $1303.7(\mathrm{~m}), 1012.5(\mathrm{~m}), 802.3$ (s), $474.4(\mathrm{~s}), 453.9$ (w) (图 S8).

\section{2 晶体结构表征}

单晶数据利用牛津单晶衍射仪收集, 光源为钼靶, 化合物 $\mathbf{1} \cdot \mathrm{H}_{2} \mathrm{O}$ 测试温度为 $100 \mathrm{~K}$ 和 $293 \mathrm{~K}$, 化合物 $\mathbf{1}$ 的 测试温度为 $173 \mathrm{~K}$ 和 $293 \mathrm{~K}$. 使用 CrysAlis Red 程序进 行单晶数据的还原和吸收校正. 在 $O L E X 2$ 软件中使用 ShelXS 或 ShelXT 程序解出粗结构, 使用 ShelXL 程序(最 小二乘法, least-squares fit on $F^{2}$ ) 对所有非氢原子进行各 向异性精修和结构精修 ${ }^{[33-35]}$. 结构中的氢原子均通过理 论加氢实现. 化合物 $\mathbf{1} \cdot \mathrm{H}_{2} \mathrm{O}$ 和 $\mathbf{1}$ 的单晶结构的 CCDC 号 分别为 2021462-2021465.

\section{3 物性测试表征}

采用 Wayne Kerr 公司的 6500B 型阻抗分析仪对粉 末压片样品进行介电测试, 采用 Janis 公司的恒温装置 进行控温. 利用 Quantum Design 公司的 XL-7 型磁学测 试系统进行磁性测试. 使用实验仪器为 Rigaku Vltima IV 型粉末 $X$ 射线衍射仪进行 $X$ 射线粉末衍射测试, 使 用条件为: $\mathrm{Cu}-\mathrm{K} \alpha$ 靶, $\lambda=0.154056 \mathrm{~nm}$, 管电流为 $15 \mathrm{~mA}$, 管电压为 $30 \mathrm{kV}$, 以石墨单色器滤波, 扫描速度为 10 $\left(^{\circ}\right) \cdot \mathrm{min}^{-1}$. 使用实验仪器 Thermal Analyzer SDT Q600, 在空气气氛中, 以 $10 \mathrm{~K} \cdot \mathrm{min}^{-1}$ 的扫描速率, 测试至 $1000{ }^{\circ} \mathrm{C}$ 的条件下进行化合物热重测试.

\section{References}

[1] Matsuoka, S.; Sugiyama, S.; Matsuoka, D.; Hirse, M.; Lethu, S.; Ano, H.; Hara, T.; Ichihara, O.; Kimura, S. R.; Murakami, S. Angew. Chem. Int. Ed. 2015, 127, 1528.

[2] Hall, J. P.; Sanchez-weatherby, J.; Alberti, C.; Quimper, C. H.; O'Sullivan, K.; Brazier, J. A.; Winter, G.; Sorensen, T.; Kelly, J. M.; Cardin, D. J.; Cardin, C. J. J. Am. Chem. Soc. 2014, 136, 17505.

[3] Mitusińska, K.; Magdziarz, T.; Bzówka, M.; Stańczak, A.; Góra, A. Biomolecules 2018, 8, 143 .

[4] Mitusińska, K.; Raczyńska, A.; Bzówka, M.; Bagrowska, W.; Góra, A. Comput. Struct. Biotechnol. J. 2020, 18, 355.

[5] Darby, J. F.; Hopkins, A. P.; Shimizu, S.; Roberts, S. M.; Brannigan,
J. A.; Turkenburg, J. P.; Thomas, G. H.; Hubbard, R. E.; Fischer, M. J. Am. Chem. Soc. 2019, 141, 15818.

[6] Zhang, J. P.; Liao, P. Q.; Zhou, H. L.; Lin, R. B.; Chen, X. M. Chem. Soc. Rev. 2014, 43, 5789.

[7] Zhang, W. X.; Xue, W.; Chen, X. M. Inorg. Chem. 2011, 50, 309.

[8] Bhattacharya, S.; Bhattacharyya, A. J.; Natarajan, S. Inorg. Chem. 2015, 54, 1254.

[9] Konavarapu, S. K.; Goswami, A.; Kumar, A. G.; Banerjee, S.; Biradha, K. Inorg. Chem. Front. 2019, 6, 184.

[10] Tang, B. Z. Acta Phys.-Chim. Sin. 2020, 36, 2001037. (唐本忠, 物 理化学学报, 2020, 36, 2001037.)

[11] Wang, X. P.; Chen, W. M.; Qi, H.; Li, X. Y.; Rajnák, C.; Feng, Z. Y.; Kurmoo, M.; Boča, R.; Jia, C. J.; Tung, C. H.; Sun, D. Chem. Eur. J. 2017, 23, 7990.

[12] Omoto, K.; Nakae, T.; Nishio, M.; Yamanoi, Y.; Kasai, H.; Nishibori, E.; Mashimo, T.; Seki, T.; Ito, H.; Nakamura, K.; Kobayashi, N.; Nakayama, N.; Goto, H.; Nishihara, H. J. Am. Chem. Soc. 2020, 142, 12651.

[13] Fernández-Navarro, L.; Iturrospe, A.; Reinoso, S.; Artetxe, B.; Ruiz-Bilbao, E.; Felices, L. S.; Gutiérrez-Zorrilla, J. M. Cryst. Growth Des. 2020, 20, 3499.

[14] Pisačić, M.; Kodrin, I.; Matijaković, N.; Chatterjee, N.; Oliver, C. L.; Kukovec, B. M.; Đaković, M. Cryst. Growth Des. 2020, 20, 401.

[15] Wang, L. F.; Zhuang, W. M.; Huang, G. Z.; Chen, Y. C.; Qiu, J. Z.; Ni, Z. P.; Tong, M. L. Chem. Sci. 2019, 10, 7496.

[16] Ge, C.; Liu, J.; Ye, X.; Han, Q. X.; Zhang, L. L.; Cui, S. Y.; Guo, Q.; Liu, G. F.; Liu, Y.; Tao, X. T. J. Phys. Chem. C 2018, 122, 15744.

[17] Wu, X. W.; Pan, F.; Yin, S.; Ge, J. Y.; Jin, G. X.; Wang, P.; Ma, J. P. CrystEngComm 2018, 20, 3955.

[18] Jin, M.; Sumitani, T.; Sato, H.; Seki, T.; Ito, H. J. Am. Chem. Soc. 2018, 140, 2875.

[19] Wang, H. Y.; Su, J.; Ma, J. P.; Yu, F.; Leong, C. F.; Deanna, M. D.; D’Alessandro, D. M.; Kurmoo, M.; Zuo, J. L. Inorg. Chem. 2019, $58,8657$.

[20] Ge, J. Y.; Chen, Z. Y.; Zhang, L.; Liang, X.; Su, J.; Kurmoo, M.; Zuo, J. L. Angew. Chem. Int. Ed. 2019, 58, 8789.

[21] Liu, J. L.; Liu, W.; Huang, G. Z.; Tong, M. L. Sci. Bull. 2015, 60, 447.

[22] Li, J.; Yang, L.; Yin, L.; Jiang, S. D.; OuYang, Z. W.; Zhang, Y. Q.; Wang, Z. X.; Song, Y. J. Inorg. Chem. 2020, 36, 1123. (李晶, 杨莉. 尹磊, 蒋尚达, 欧阳钟文, 张义权, 王振兴, 宋友, 无机化学学 报, 2020, 36, 1123.)

[23] Zhao, J. P.; Wang, W. W.; Han, S. D; Li, Q. W.; Li, N.; Liu, F. C.; Bu, X. H. Acta Chim. Sinica doi: 10.6023/A20080341. (赵畑鹏, 王 玮玮, 韩松德, 李泉文, 李娜, 刘福臣, 卜显和, 化学学报, doi: 10.6023/A20080341)

[24] Zhao, H. X.; Liu, J. X.; Long, L. S.; Bokov, A. A.; Ye, Z. G.; Huang, R. B.; Zheng, L. S. J. Phys. Chem. C 2012, 116, 14199.

[25] Xu, H. R.; Zhang, Q. C.; Ren, Y. P.; Zhao, H. X.; Long, L. S.; Huang, R. B.; Zheng, L. S. CrystEngComm 2011, 13, 6361.

[26] Coronado, E.; Galán-Mascarós, J. R.; Gómez-García, C. J.; MartíGastaldo, C. Inorg. Chem. 2005, 44, 6197.

[27] Asaji, T.; Ashitomi, K. J. Phys. Chem. C 2013, 117, 10185.

[28] Mączka, M.; Gągor, A.; Macalik, B.; Pikul, A.; Ptak, M.; Hanuza, J. Inorg. Chem. 2014, 53, 457.

[29] Mączka, M.; Kadłubański, P.; Freire, P. T. C.; Macalik, B.; Paraguassu, W.; Hermanowicz, K.; Hanuza, J. Inorg. Chem. 2014, 53, 9615.

[30] Armentano, D.; Mastropietro, T. F.; Munno, G. D.; Rossi, P.; Lloret, F.; Julve, M. Inorg. Chem. 2008, 47, 3772.

[31] Fisher, M. E. Am. J. Phys. 1964, 32, 343.

[32] Demeshko, S.; Leibeling, G.; Dechert, S.; Fuchs, S.; Pruschke, T.; Meyer, F. ChemPhysChem 2007, 8, 405.

[33] Dolomanov, O. V.; Bourhis, L. J.; Gildea, R. J.; Howard, J. A. K.; Puschmann, H. J. Appl. Cryst. 2009, 42, 339.

[34] Sheldrick, G. M. Acta Cryst. 2008, A64, 112.

[35] Sheldrick, G. M. Acta Cryst. 2015, C71, 3.

(Cheng, B.) 